\title{
Stability Analysis of Periodic Solutions of Some Duffing's Equations
}

\author{
E. O. Eze, U. E. Obasi, E. U. Agwu \\ Department of Mathematics, Michael Okpara University of Agriculture, Umudike, Umuahia, Nigeria \\ Email: obinwanneeze@gmail.com, sirurchobasi@gmail.com, agwuemekauchendu@gmail.com
}

How to cite this paper: Eze, E.O., Obasi, U.E. and Agwu, E.U. (2019) Stability Analysis of Periodic Solutions of Some Duffing's Equations. Open Journal of Applied Sciences, 9, 198-214.

https://doi.org/10.4236/ojapps.2019.94017

Received: October 10, 2018

Accepted: April 20, 2019

Published: April 23, 2019

Copyright $\odot 2019$ by author(s) and Scientific Research Publishing Inc. This work is licensed under the Creative Commons Attribution International License (CC BY 4.0).

http://creativecommons.org/licenses/by/4.0/

\begin{abstract}
In this paper, some stability results were reviewed. A suitable and complete Lyapunov function for the hard spring model was constructed using the Cartwright method. This approach was compared with the existing results which confirmed a superior global stability result. Our contribution relies on its application to high damping door constructions. (2010 Mathematics Subject Classification: 34B15, 34C15, 34C25, 34K13.)
\end{abstract}

\section{Keywords}

Stability Analysis, Duffing's Equations, Periodic Solutions, MATCAD, Cartwright Method

\section{Introduction}

In real life, most problems that occur are non-linear in nature and may not have analytic solutions except by approximations or simulations and so trying to find an explicit solution may in general be complicated and sometimes impossible. Duffing's equation is a second order non-linear differential equation used to model such problems of non-linear nature [1]. In particular, it is used to model damped and driven oscillators, for example, modelling of the brain [2], in prediction of earthquake occurrences [3], signal processing [4] and crash analysis [5]. In [6], differential equation which describes a non-linear oscillation was first introduced by Duffing's with cubic stiffness constant. The general form of Duffing's equation is:

$$
\ddot{x}+c \dot{x}+g(t, x)=p(t)
$$

where $p(t)$ is continuous and $2 \pi$-periodic in $t \in \mathbb{R}$.

The study of periodic solution of Duffing's equation is like that of the study of classical Hamiltonian equation of motion which is characterized by multiplicity 
of periodic solution. Various techniques for investigating the stability of periodic solutions of Duffing's equation have been reported, (see [7] [8] [9] [10] [11]). Our approach to study stability of periodic solutions is by the use of the Lyapunov direct method. Many researchers have obtained useful and valid results using the Lyapunov second method (direct method) for stability analysis and construction of appropriate Lyapunov functions for other Duffing equation but the use and construction of Lyapunov function using Cartwright method are rare in literature, for instance [12] [13] [14] [15] [16]. The application of this method is in constructing a scalar function and its derivatives with peculiar characterizations. When these characterizations are satisfied, the stability behaviour of the system is solved. The difficulties in the construction of suitable Lyapunov functions in nonlinear systems have attracted the attention of many researchers and have been summarized in [17] [18] [19] that discussed the general approach to the stability study of periodic solution which is related to the classical Lyapunov theorem based on linear approximations. This reduces the stability study of periodic solutions to the stability of the system linearized at the periodic motion. Since linearized systems contain periodic coefficients, the theory of parametric resonance can be applied. Such approach with the analysis of Floquet multipler is used in [9] [20] [21]. The other traditional approach to the study of stability of periodic solutions is the approximate average and multiple scales method, which reduces original time dependent dyamical systems to autonomous system. In this case stability study is the analysis of fixed point, (see [22]).

Our construction of suitable Lyapunov function rests squarely on the approach of [23]. Others who used this approach are [16] [24]. Very few nonlinear systems can be solved explicitly and so we must rely on numerical schemes to approximate the solutions, (see [25] [26] [27] [28]). This paper is motivated by studying [11] [29] where stability analysis of the unforced and damped cubic-quintic Duffing oscillator of the form

$$
\ddot{u}+\delta \dot{u}+\alpha u+\beta u^{3}+\mu u^{5}=0
$$

was carried out. Using Equation (1.2) the existence of a three term valid solution was obtained using derivative expansion method. The derivative expansion method is one of the perturbative methods which require the existence of a small parameter and is therefore not valid in principle for the Duffing equation in which the nonlinearity is large. Its stability analysis of the equilibrium point was carried out using the eigenvalue approach. Secondly, our motivation was augmented by reading the works of [10] where new criteria for existence and asymptotic stability of periodic solutions of a Duffing equation of the form

$$
\ddot{x}+c \dot{x}+g(t, x)=0
$$

were derived. This approach exposed by [10] is useful when the non-linearity does not admit the decomposition of $g(t, x)=g(x)+h(t)$. In line with the above review and ongoing research in this direction, the objective of this paper is to investigate the stability analysis of periodic solutions of the Duffing type 


$$
\ddot{x}+c \dot{x}+a x+b x^{2}+2 x^{3}=h(t)
$$

with boundary conditions as:

$$
\begin{aligned}
& x(0)=x(2 \pi) \\
& \dot{x}(0)=\dot{x}(2 \pi)
\end{aligned}
$$

where $a, b, c$ are real constants and $h:[0,2 \pi] \rightarrow \mathbb{R}^{\mathfrak{N}}$ is continuous. In Equation (1.4), $a$ is the stiffness constant, $c$ is the coefficient of viscous damping and $b x^{2}+2 x^{3}$ represents the nonlinearity in the restoring force acting like a hard spring. Equation (1.4) has received wide interest in neurology, modelling of mechanical systems such as shock absorbers in most vehicles. It can be used to model plant stems to better understand the effect of nonlinear stiffness or resonant behavior of plants. It is a model arising in many branches of Physics and Engineering such as oscillation of rigid pendulum using moderately large amplitude motion [29], Vibration of buckled beam [30] etc. This equation together with Van der Pol's has been reported in textbooks as examples of nonlinear oscillation. See [31] [32] [33].

\section{Preliminaries}

\section{Definition 2.1. (Properties of Lyapunov Function)}

The Lyapunov function has the following properties:

a) Continuity: $V(t, X)$ is continuous and single valued under the condition $t \geq T$ and $\left|x_{i}\right|<H$ and $V(t, 0) \equiv 0$.

b) $V(t, X)$ is positive definite; and

c) $\dot{V}=\frac{\partial V}{\partial x_{1}} \dot{x}_{1}+\frac{\partial V}{\partial x_{2}} \dot{x}_{2}+\cdots+\frac{\partial V}{\partial x_{n}} \dot{x}_{n}+\frac{\partial V}{\partial t}$, representing the total derivative with respect to $t$ is negative definite.

\section{Definition 2.2. (Complete Lyapunov function)}

A Lyapunov function $V$ defined as $V: I \times \mathbb{R}^{n} \rightarrow \mathbb{R}$ is said to be COMPLETE if for $X \in \mathbb{R}^{n}$,

1) $V(t, X) \geq 0$

2) $V(t, X)=0$ if and only if $X=0$ and

3) $\dot{V}(t, X) \leq-c|X|$ where $c$ is any positive constant and $|X|$ given by

$$
|X|=\left(\sum_{i=1}^{n}\left(x_{i}^{2}\right)\right)^{\frac{1}{2}} \rightarrow \infty
$$

It is INCOMPLETE if 3 ) is not satisfied.

Theorem 2.3. Consider a system of differential equations

$$
\dot{X}=f(x)
$$

$f: D \rightarrow \mathbb{R}^{2}$. Let $X=0$ be an equilibrium point of Equation (1.6).

Let $V: D \rightarrow \mathbb{R}$ be a continuously differentiable function; such that:

1) $V(0)=0$;

2) $V(x)>0$ in $D-\{0\}$; 
3) $\dot{V}(x) \leq 0$ in $D-\{0\}$.

Then $X=0$ is stable.

Theorem 2.4. Let $V: D \rightarrow \mathbb{R}$ be a continuously differentiable function such that:

1) $V(0)=0$;

2) $V(x)>0$ in $D-\{0\}$;

3) $\dot{V}(x)<0$ in $D-\{0\}$.

Then $X=0$ is "asymptotically stable".

Theorem 2.5. Let $V: D \rightarrow \mathbb{R}$ be a continuously differentiable function such that:

1) $V(0)=0$

2) $V(x)>0$ in $D-\{0\}$;

3) $V(x)$ is "radially bounded";

4) $\dot{V}(x)<0$ in $D-\{0\}$.

Then $X=0$ is "globally asymptotically stable".

Theorem 2.6. Suppose all conditions for asymptotic stability are satisfied. In addition to it, suppose there exists constants $K_{1}, K_{2}, K_{3}, P$

1) $K_{1}\|X\|^{P} \leq V(X) \leq K_{2}\|X\|^{P}$;

2) $\dot{V}(X) \leq-K_{3}\|X\|^{P}$.

Then the origin $X=0$ is "exponentially stable".

Moreover, if this conditions hold globally, then the origin $X=0$ is globally exponentially stable.

\section{Results}

\subsection{Construction of Lyapunov Function for the Duffing Equation Using Cartwright Method}

We adapt the method of construction of Lyapunov function used in [23] and extend it to the second order non-linear differential equation of the Duffing type of the form (3.1). In the sequel, [34] asserted that Lyapunov functions are vital in determining stability, instability, boundedness and periodicity of ordinary differential. Considering the Duffing equation of the form

$$
\ddot{x}+c \dot{x}+a x+b x^{2}+2 x^{3}=p(t)
$$

The equivalent systems of Equation (1.7) is

$$
\begin{aligned}
& \dot{x}_{1}=x_{2} \\
& \dot{x}_{2}=-c x_{2}-a x_{1}-h\left(x_{1}\right)
\end{aligned}
$$

where $h\left(x_{1}\right)=b x_{1}^{2}+2 x_{1}^{3}$ and $p(t)=0$.

Writing the equivalent systems of Equation (1.7) in compact form, we have

$$
\dot{X}=A X
$$

where $A=\left[\begin{array}{cc}0 & 1 \\ -a-h(x) & -c\end{array}\right], X=\left[\begin{array}{l}x_{1} \\ x_{2}\end{array}\right]$.

The method discussed here is based on the fact that the matrix $A$ has all its ei- 
genvalues as negative real parts. Then from the general theory which corresponds to any positive quadratic form $U(x)$, there exists another positive definite quadratic form $V(x)$ such that

$$
\dot{V}=-U
$$

Choosing the most general quadratic form of order two and picking the coefficient in the quadratic form to satisfy Equation (1.10) along the solution paths of Equation (1.10) we assume $V$ to be defined by

$$
\begin{aligned}
& 2 V=A x^{2}+B y^{2}+2 K x y \\
& \dot{V}=A x \dot{x}+B y \dot{y}+K(\dot{x} y+\dot{y x}) \\
&= A x y+B(-c y-a x-h(x))+K y^{2}+K x(-c y-a x-h(x)) \\
&= A x y-B c y^{2}-B y a x-B y h(x)+K y^{2}-K c x y-K a x^{2}-K x h(x)
\end{aligned}
$$

Simplifying the coefficients we have

$$
\dot{V}=(A-B a-K c) x y+(K-B c) y^{2}-(K a) x^{2}-(K x+B y) h(x)
$$

To make $\dot{V}$ negative definite, we equate the coefficient of mixed variable to zero and the coefficients of $x^{2}$ and $y^{2}$ to any positive constant (say $\delta$ ) as outlined in Table 1

$$
\begin{gathered}
A-B a-K c=0 \\
K-B c=\delta \\
-K a=\delta \\
-(K x+B y)=0
\end{gathered}
$$

From Equation (3) above

$$
\begin{aligned}
& -K a=\delta \\
& K=-\frac{\delta}{a}
\end{aligned}
$$

Then substituting the value of $K$ into Equation (2) we obtain

$$
\begin{aligned}
& -\frac{\delta}{a}-B c=\delta \\
& -B c=\delta+\frac{\delta}{a} \\
& B=-\frac{\delta(a+1)}{c a}
\end{aligned}
$$

Substituting for $K$ and $B$ in (1) we have

$$
A=B a+K c=-\frac{\delta(a+1)}{c}-\frac{\delta c}{a}
$$

which by further simplification gives that

$$
A=-\frac{\delta}{c a}\left[(a+1) a+c^{2}\right]
$$

Substituting for the values of the constant $A, B, K$ in Equation (1.11) gives

$$
2 V=-\frac{\delta}{c a}\left[(a+1) a+c^{2}\right] x^{2}-\frac{\delta}{c a}[a+1] y^{2}-2 \frac{\delta}{a} x y
$$


Table 1. A table showing terms and coefficients of Equation (1.12).

\begin{tabular}{cc}
\hline Terms & Coefficient \\
\hline$x y$ & $A-B a-K c$ \\
$y^{2}$ & $K-B c$ \\
$x^{2}$ & $-K a$ \\
$h(x)$ & $-(K x+B y)$ \\
\hline
\end{tabular}

$$
V=-\frac{\delta}{2 c a}\left[\left((a+1) a+c^{2}\right) x^{2}+(a+1) y^{2}+2 c x y\right]
$$

By choosing $\frac{\delta}{c a}=-1$

$$
V(x)=\frac{1}{2}\left[\left((a+1) a+c^{2}\right) x^{2}+(a+1) y^{2}+2 c x y\right]>0
$$

Using Equation (1.19) and the fact that $\dot{V}<0$, the equilibrium point is asymptotically stable.

\subsection{Stability Analysis of Periodic Solution of Duffing Equation Using the Eigenvalue Approach}

Considering the Duffing equation of the form (1.7), the first equivalent systems of (1.7) is given by

$$
\begin{aligned}
& \dot{x}=y \\
& \dot{y}=-c y-a x-b x^{2}-2 x^{3}+h(t)
\end{aligned}
$$

For the unforced case, Equation (1.19) is reduced to

$$
\begin{aligned}
& \dot{x}=y \\
& \dot{y}=-c y-a x-b x^{2}-2 x^{3}
\end{aligned}
$$

At fixed points, $\dot{x}=y=0$.

So that $y=0$ and $\dot{y}=-a x-b x^{2}-2 x^{3}=x\left(-a x-b x-2 x^{2}\right)=0$.

Giving us $x=0, x_{1}=\frac{-b+\sqrt{b^{2}-8 a}}{4}$ and $x_{2}=\frac{-b-\sqrt{b^{2}-8 a}}{4}$ which correspond to $(0,0),\left(x_{1}, 0\right)$ and $\left(x_{2}, 0\right)$ at fixed point. Analysis of the stability of the fixed points can be done by linearizing Equation (1.20) which gives

$$
\begin{aligned}
& \ddot{x}=\dot{y} \\
& \ddot{y}=-c \dot{y}-\left(a+2 b x+6 x^{2}\right) \dot{x}
\end{aligned}
$$

The matrix equation for (1.21) can be written as

$$
\left[\begin{array}{l}
\ddot{x} \\
\ddot{y}
\end{array}\right]=\left[\begin{array}{cc}
0 & 1 \\
-\left(a+2 b x+6 x^{2}\right) & -c
\end{array}\right]\left[\begin{array}{l}
\dot{x} \\
\dot{y}
\end{array}\right]
$$

Examining the stability at the point $(0,0)$ gives 


$$
\begin{gathered}
{\left[\begin{array}{cc}
0-\lambda & 1 \\
-\left(a+2 b x+6 x^{2}\right)-\lambda & -c-\lambda
\end{array}\right]} \\
\lambda c+\lambda^{2}+a+2 b x+6 x^{2}+\lambda=0 \\
\lambda^{2}+\lambda(1+c)+g=0 \\
\lambda=\frac{-(1+c) \pm \sqrt{(1+c)^{2}-4 g}}{2}
\end{gathered}
$$

where $\lambda_{1}=\frac{1}{2}\left(-(1+c)+\sqrt{(1+c)^{2}-4 g}\right)$ and $\lambda_{2}=\frac{1}{2}\left(-(1+c)-\sqrt{(1+c)^{2}-4 g}\right)$ are the roots of Equation (1.23). $\lambda_{1}$ and $\lambda_{2}$ can be written as

$$
\begin{gathered}
\lambda_{1}=\frac{1}{2}\left(-\delta+\sqrt{\delta^{2}-4 g}\right) \text { and } \lambda_{2}=\frac{1}{2}\left(-\delta-\sqrt{\delta^{2}-4 g}\right) \text { where } \delta=1+c \text { and } \\
g=a+2 b x+6 x^{2}
\end{gathered}
$$

The coefficient of $\beta$ shows that the Duffing equation is highly damped representing a hard spring. This hard spring is represented in Equation (1.21) where the coefficient of $x^{2}$ is 6 .

At the origin, $\lambda_{ \pm}^{(0,0)}=\frac{1}{2}\left(-\delta \pm \sqrt{\delta^{2}-4 g}\right)$.

With $\delta=0, \quad \lambda_{1,2}=\frac{1}{2}( \pm \sqrt{-4 g})$.

For this, we consider the following cases:

1) When $g=0, \lambda_{1,2}=0$ and this implies that $b, x, a$ are all zero;

2) When $g>0, \lambda_{1,2}= \pm i \sqrt{g}$ which corresponds to critical points that are centres for which stability is ensured;

3) When $g<0, \lambda_{1,2}= \pm \sqrt{g}$ which corresponds to saddles giving rise to instability [11].

With $\delta>0, \lambda_{1,2}=\frac{1}{2}\left(-\delta \pm \sqrt{\delta^{2}-4 g}\right)$.

For this, we consider the following cases:

1) When $g=0, \lambda_{1,2}=0,-\delta$;

2) When $g>0, \lambda_{1,2}=\frac{1}{2}\left(-\delta \pm \sqrt{\delta^{2}-4 g}\right)$.

For the discriminate, we have the following cases:

When $\delta^{2}<4 g, \lambda_{1,2}=\frac{1}{2}\left(-\delta \pm i \sqrt{\delta^{2}-4 g}\right)$ shows that the roots are imaginary which to lead to spiral and asymptotic stability.

When $\delta^{2}>4 g, \quad \lambda_{1,2}=\frac{1}{2}\left(-\delta \pm \sqrt{\delta^{2}-4 g}\right)$ shows that the roots are real which leads to saddles and instability.

When $\delta^{2}=4 g, \lambda_{1,2}= \pm \sqrt{g}$ shows that the roots are real corresponding to saddles and instability.

When $g<0, \lambda_{1,2}=\frac{1}{2}\left(\delta \pm \sqrt{\delta^{2}+4 g}\right)$. 
For the discriminate, we consider the following cases:

1) When $\delta^{2}+4 g<0, \quad \lambda_{1,2}=\frac{1}{2}\left(\delta \pm i \sqrt{\delta^{2}+4 g}\right)$ which corresponds to spirals and asymptotic stability.

2) When $\delta^{2}+4 g>0, \quad \lambda_{1,2}=\frac{1}{2}\left(\delta \pm i \sqrt{\delta^{2}+4 g}\right)$ which leads to instability.

3) When $\delta^{2}+4 g=0, \lambda_{1,2}= \pm(i \sqrt{g})$ which leads to centres and instability.

Interestingly, for special case when $c=0$ with no forcing term equation (1.20) becomes

$$
\begin{gathered}
\dot{x}=y \\
\dot{y}=-a x-b x^{2}-2 x^{3}
\end{gathered}
$$

The above can be integrated by quadrature, differentiating (1.25) and plugging in (1.26) gives

$$
\dot{x}=\dot{y}=-a x-b x^{2}-2 x^{3}
$$

Multiplying both sides by $\dot{x}$ gives

$$
\dot{x} \dot{x}-a x \dot{x}-b \dot{x} x^{2}-2 \dot{x} x^{3}=0
$$

Equation (1.28) can be written as

$$
\frac{\mathrm{d}}{\mathrm{d} t}\left[\frac{1}{2} \dot{x}^{2}-\frac{1}{2} a x^{2}-\frac{1}{3} b x^{3}-\frac{1}{2} x^{4}\right]=0
$$

So we have a variant of motion

$$
h=\frac{1}{2} \dot{x}^{2}-\frac{1}{2} a x^{2}-\frac{1}{3} b x^{3}-\frac{1}{2} x^{4}
$$

solving for $\dot{x}^{2}$ gives $\dot{x}^{2}=\left(\frac{\mathrm{d} x}{\mathrm{~d} t}\right)^{2}=2 h+a x^{2}+\frac{2}{3} b x^{3}+x^{4}$

$$
\begin{array}{r}
\frac{\mathrm{d} x}{\mathrm{~d} t}=\sqrt{2 h+a x^{2}+\frac{2}{3} b x^{3}+x^{4}} \\
t=\int \mathrm{d} t=\int \frac{\mathrm{d} t}{\sqrt{2 h+a x^{2}+\frac{2}{3} b x^{3}+x^{4}}}
\end{array}
$$

Note that the invariant of motion $h$ satisfies $\dot{x}=\frac{\partial h}{\partial \dot{x}}=\frac{\partial h}{\partial y}$ where

$$
\frac{\partial h}{\partial x}=a x+b x^{2}+2 x^{3}=\dot{y}
$$

So the equation of Duffing oscillator are given by the Hamiltonian system

$$
\dot{x}=\frac{\partial h}{\partial y} \text { and } \dot{y}=-\frac{\partial h}{\partial x}
$$

We consider the stability analysis of Duffing's equation for different values of $a, b, c$, as illustrated in Table 2 . 
Table 2 . The stability analysis and numerical solutions of duffing's equation at different values $a, b, c$.

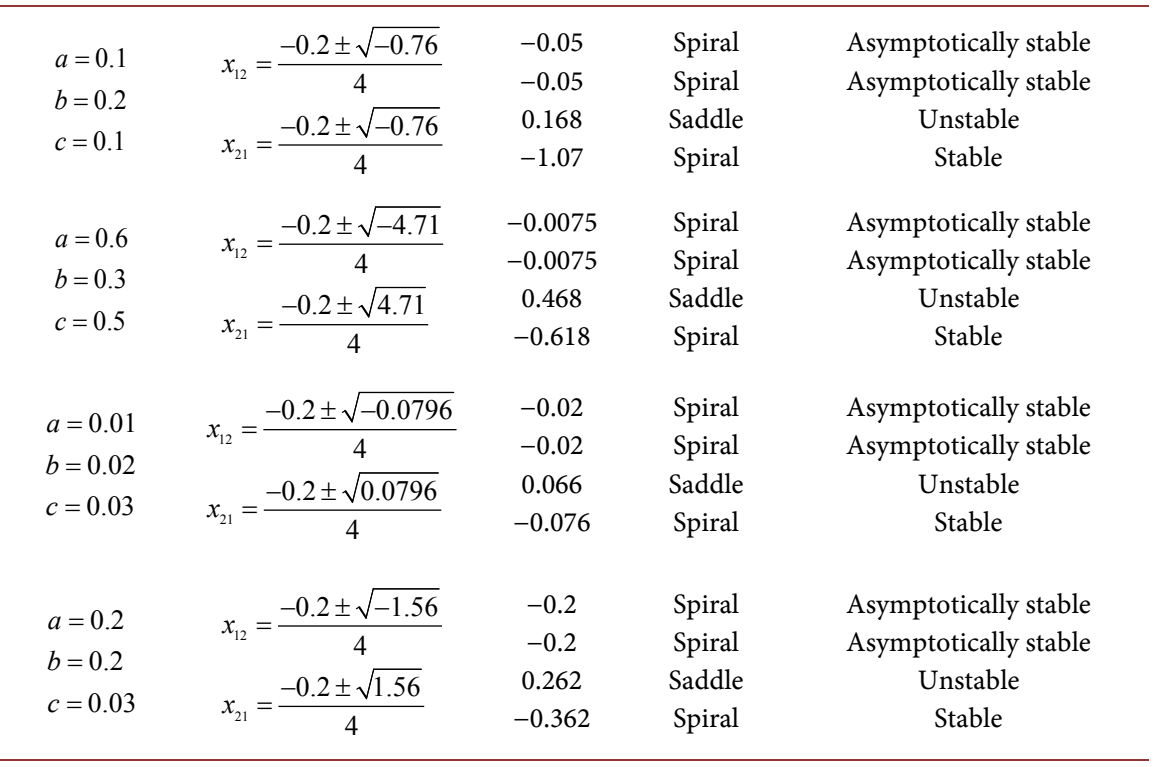

Field work 2017.

\subsection{Numerical Solution of Duffing's Equation Using Mathcad}

$a:=0.1 \quad b:=0.2 \quad c:=0.1$

$t_{0}:=0 \quad t_{1}:=150$ Solution interval endpoints;

ic $:=\left[\begin{array}{l}0 \\ 1\end{array}\right]$ Initial condition vector;

$N:=1500$ Number of solution values on $\left[t_{0}, t_{1}\right]$.

$D(t, X):=\left[\begin{array}{c}X_{1} \\ -a \cdot X_{0}-b \cdot\left(X_{0}\right)^{2}-2 \cdot\left(X_{0}\right)^{3}-c \cdot X_{1}\end{array}\right]$ Derivative function.

$S:=\operatorname{rkfixed}\left(i c, t_{0}, t_{1}, N, D\right)$.

$T:=S^{\langle 0\rangle}$ Independent variable values.

$X_{1}:=S^{\langle 1\rangle}$ Solution function values.

$X_{2}:=S^{\langle 2\rangle}$ Derivative function values.

\subsection{Stability Analysis of Duffing Equation under Oyesanya and Nwamba (2013)}

The unforced and damped cubic-quintic Duffing oscillator is given by

$$
\ddot{u}+\delta \dot{u}+\alpha u+\beta u^{3}+\mu u^{5}=0
$$

From Equation (1.32) we obtain the autonomous dynamical system

$$
\left.\begin{array}{l}
y=\dot{u} \\
\dot{y}=-\delta \dot{u}-\alpha u-\beta u^{3}-\mu u^{5}
\end{array}\right\}
$$

From the condition $\dot{u}=0$ we obtain $y=0$ which gives us

$$
\alpha u+\beta u^{3}+\mu u^{5}=0
$$


Equation (1.34) can be written as

$$
u\left[\alpha+\beta u^{2}+\mu u^{4}\right]=0
$$

We obtain the roots of (1.35) as

$$
u_{0}=0, u_{1,2,3,4}= \pm \sqrt{\frac{1}{2 \mu}\left[-\beta \pm \sqrt{\beta^{2}-4 \mu \alpha}\right]}
$$

Then the equation satisfied by the eigenvalues of our systems stability matrix is

$$
\lambda^{2}+\delta \lambda=-\alpha-3 \beta u^{2}-5 \mu u^{4}
$$

where $\underline{u}$ denotes $u_{1} u_{2} u_{3}$ and $u_{4}$ or the $u$-coordinate of an equilibrium point. Whether our eigenvalues will be complex, real or imaginary will be determined by the values of $\delta$ and $g=5 \mu u^{4}+3 \beta u^{2}+\alpha \quad$ with $=0, \quad \lambda_{1,2}=\frac{1}{2}[ \pm \sqrt{-(4 g)}]$. For this we consider the following cases:

1) $g=0$ : for this case $\lambda_{1,2}=0$.

This contradicts our assumption that det $J \neq 0$ and it also implies that $\alpha, \beta, \mu$ are all zero.

2) $g>0:$ for this case $\lambda_{1,2}= \pm i \sqrt{g}$.

This corresponds to critical points that are centers for which stability is ensured.

3) $g<0$ : for this case $\lambda_{1,2}= \pm \sqrt{g}$ which corresponds to saddles giving rise to instability in (1.35) with $\delta>0$,

$$
\lambda_{1,2}=\frac{1}{2}\left[-\delta \pm \sqrt{\delta^{2}-4 g}\right]
$$

Then considering the case below we have:

1) $g=0$ : giving the values $\lambda_{1,2}=0,-\delta$ which goes contrary to our aassumption that det $j \neq 0$. It also implies that $\alpha, \beta, \mu$ are all zero.

2) $g>0$ : for this case

$$
\lambda_{1,2}=\frac{1}{2}\left[-\delta \pm \sqrt{\delta^{2}-4 g}\right]
$$

Considering the discriminant fot this case, we have three cases.

a) $\delta^{2}<4 g, \lambda_{1,2}=\frac{1}{2}\left[-\delta \pm \sqrt{\delta^{2}-4 g}\right]$ which corresponds to spirals and asymptotic stability.

b) The case $\delta^{2}>4 g$ gives the values of the form $\lambda_{1,2}=\frac{1}{2}[-\delta \pm \sqrt{p}], p>0$ which corresponds to nodes resulting in asymptotic stability if $\delta>\sqrt{p}$ and to saddles and consequently instability if $\delta<\sqrt{p}$.

c) $\delta^{2}=4 g$ gives $\lambda_{1,2}= \pm \sqrt{g}$. For this case we have saddles and hence instability.

3) $g<0$ leads us to have $\lambda_{1,2}=\frac{1}{2}\left[\delta \pm \sqrt{\delta^{2}-4 g}\right]$.

Considering the discriminate we consider the three cases as follows: 
a) $\delta^{2}+4 g<0:$ this case gives $\lambda_{1,2}=\frac{1}{2}\left[\delta \pm i \sqrt{\delta^{2}-4 g}\right]$ and we have spirals and consequently asymptotic stability.

b) $\delta^{2}+4 g>0$ gives $\lambda_{1,2}=\frac{1}{2}[\delta \pm i \sqrt{Q}], Q>0$ and this leads us to the existence of node and instability if $\delta>\sqrt{Q}$ or saddles and instability if $\delta<\sqrt{Q}$.

c) $\delta^{2}+4 g=0, \lambda_{1,2}= \pm[i \sqrt{g}]$ which yield centers and stability.

We now consider the stability analysis of the dynamics for a few choices of $\alpha, \beta, \mu$ and $\delta$ by using employing Equations (1.35) and (1.36). These are illustrated in Table 3.

\subsection{Stability Analysis of Torres (2004)}

We consider the Duffing's equation of the form

$$
\ddot{x}+c \dot{x}+g(t, x)=0
$$

with $c>0$ and $g:[0,2 \pi] \times R \rightarrow R$ is a $L^{1}$-caratheodory function such that the partial derivative $g_{x}$ exists and it is:

$L^{1}$-caratheodory. For a given $1 \leq p \leq+\infty$, let us define the set

$$
\Omega_{p, c}=\left\{a \in L^{p}(0,2 \pi): a>0,\|a\|_{p}<\left(1+\frac{c^{2}}{4}\right) k\left(2 p^{*}\right)\right\}
$$

with $p^{*}, k$ defined. Let $\varphi$ be a $2 \pi$ periodic solution of Equation (1.37), we

Table 3. Stability analysis of the dynamics of the oscillator for few choices of parameter $\alpha$, $\beta$ and $\mu$.

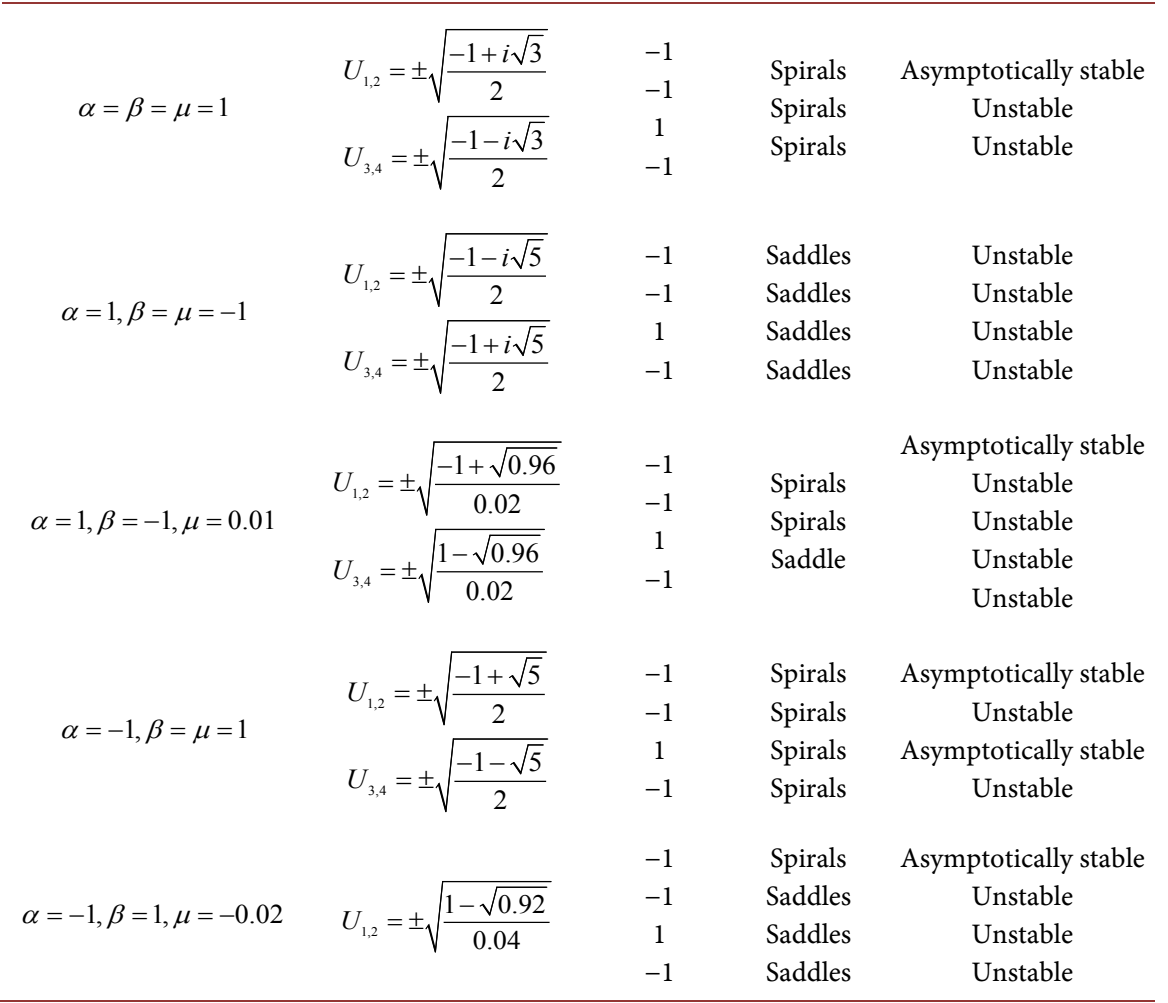


assume that $a \in \Omega_{p, c}$ exists such that

$$
g_{x}(t, \varphi(x))<a(t) \text { for a.e } t \in[0,2 \pi]
$$

For such an a, the operator $L$ as defined in maximum principle is inversely positive. By defining the operator.

$$
\begin{aligned}
& F: C[0,2 \pi] \rightarrow L^{1}(0,2 \pi), H: L^{1}(0,2 \pi) \rightarrow W \\
& G_{x}=a(x) \rightarrow g(x) \quad H=L^{-1} F .
\end{aligned}
$$

The solution $\varphi$ can be seen as a fixed point of the operator $H$. Next, we recall that an isolated $2 \pi$-periodic solution has a well-defined index $\gamma(\varphi)$ and $|\gamma(\varphi) \leq 1|$.

\section{Discussion}

Figure 1 is the MATCAD showing the behavior of the Duffing's equation when $a=0.1, b=0.2$ and $c=0.1$ is periodic. We observe asymptotically stable at both saddle and spiral point i.e. $\left(\frac{-0.2 \pm \sqrt{-0.76}}{4}, 0\right)$ which are three equilibrium points observed. This situation is seen in Duffing's equation.

Figure 2 shows the dynamics of Duffing's equation when $a=0.6, b=0.3, c=0.5$. We observed asymptotically stable at spiral point i.e. $\left(\frac{-0.3 \pm \sqrt{-4.71}}{4}, 0\right)$. This shows that the phase is revolving round it. At (0.468) the point was saddle showing that it is unstable.

Figure 3: the MATCAD shows the dynamics of the Duffing's equation when $a=0.01, b=0.02, c=0.03$. Three equilibrium points was observed. Saddles were obtained at $(-0.02,0.066)$ showing that the phases are toward the origin.

Figure 4: the MATCAD were obtained for the values of $a=-0.2, b=0.2, c=0.03$. In this case it is important to note that the phase line tend to converge toward the equilibrium points.

Figure 5: The trajectory profile of Duffing's equation were obtained for the values $a=-0.2, b=0.2, c=0.03$. In this case, there is an increase in oscillation which is as a result of decrease in the damping coefficient. That is decrease in damping leads to increase in oscillation.

Figure 6: The phase portrait of Duffing's equation was obtained. We observed that the phase line was depicting a center of non-stable node.

Figure 7: The trajectory profile of Duffing's equation were obtained for the values $a=0.6, b=0.3, c=0.5$. In this case, there is a decrease in the maximum displacement from the origin which leads to decrease in oscillation. The decrease in oscillation is as a result of increase in the damping coefficient. That is increase in damping leads to decrease in oscillation.

Figure 8: In this case, the phase portrait was depicting the center as an unstable node.

\section{Conclusion}

The study of the stability analysis of periodic solution using Lyapunov direct 


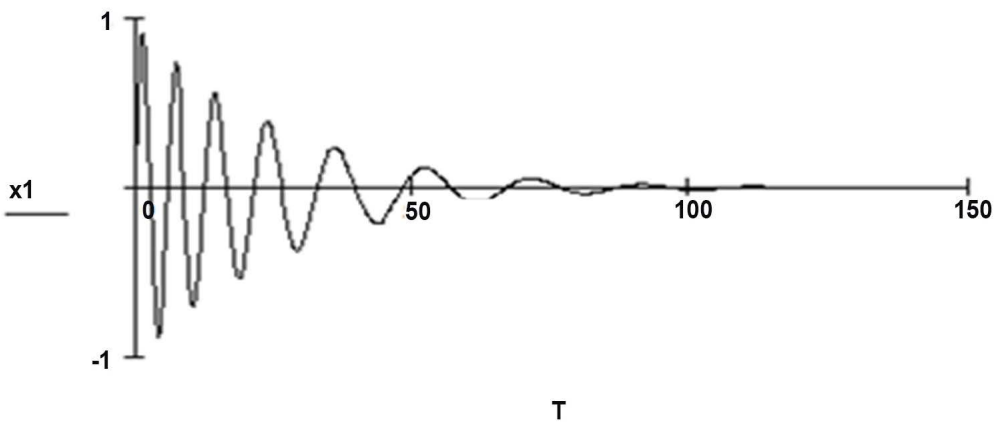

Figure 1. Trajectory profile of Duffing equation for values $a=0.1, b=0.2, c=0.1$.

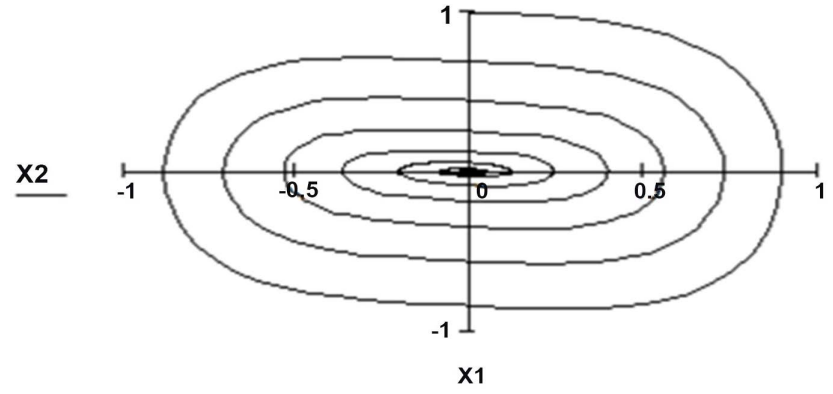

$$
a:=0.6 \quad b:=0.3 \quad c:=0.5
$$

Figure 2. Phase portrait of Duffing's equation showing asymptotic stability of solution as a spiral sink.

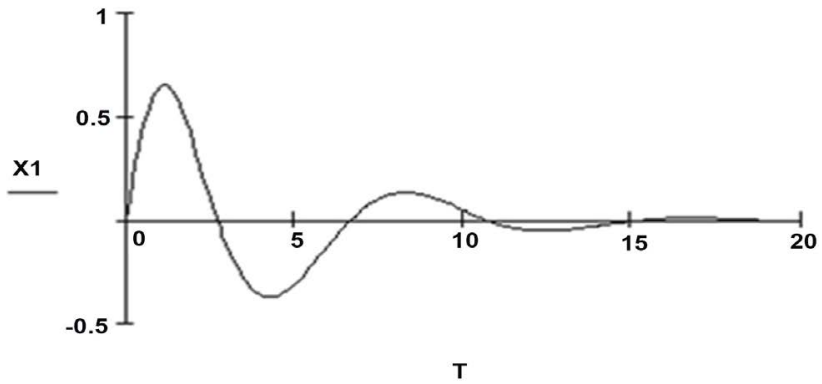

Figure 3. Trajectory profile of Duffing equation for values $a=0.6, b=0.3, c=0.5$.

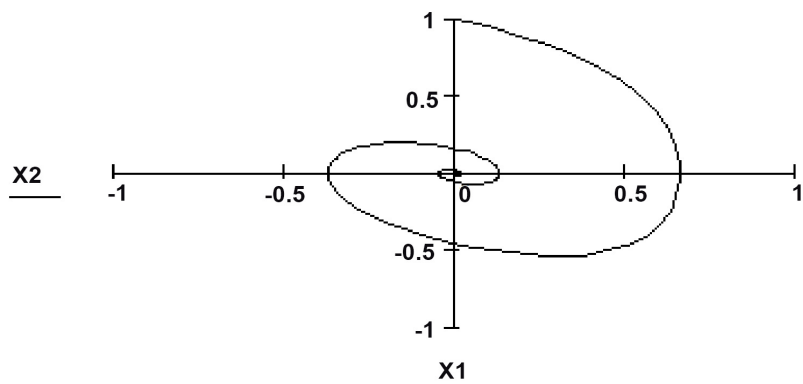

$$
a:=0.01 \quad b:=0.02 \quad c:=0.03
$$

Figure 4. Phase portrait of Duffing's equation depicting asymptotic stability of solution as a spiral sink. 


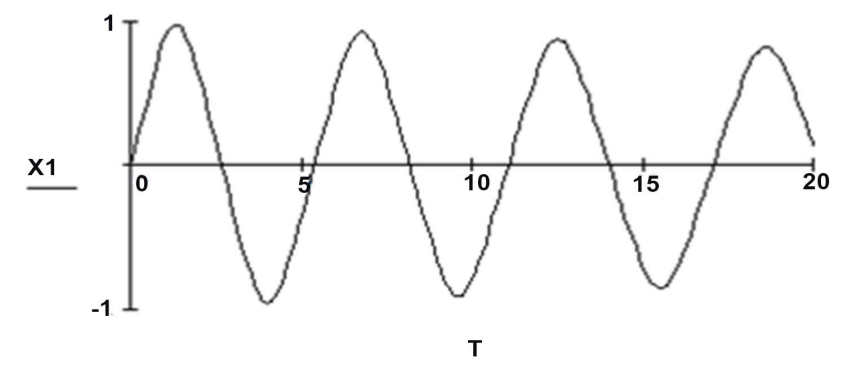

Figure 5. Oscillatory profile of Duffing equation for parameters $a=-0.2, b=0.2, c=0.03$.

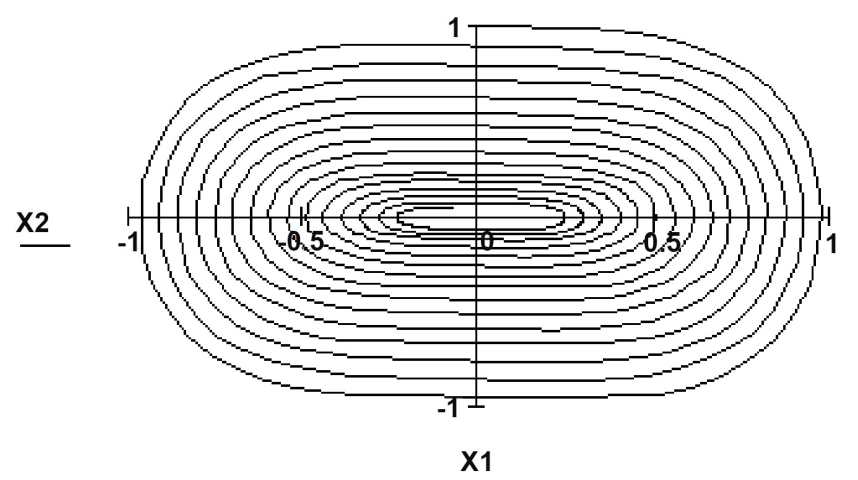

$$
a:=-0.2 \quad b:=0.2 \quad c:=0.03
$$

Figure 6. Phase portrait of Duffing's equation depicting a centre of a non-stable node.

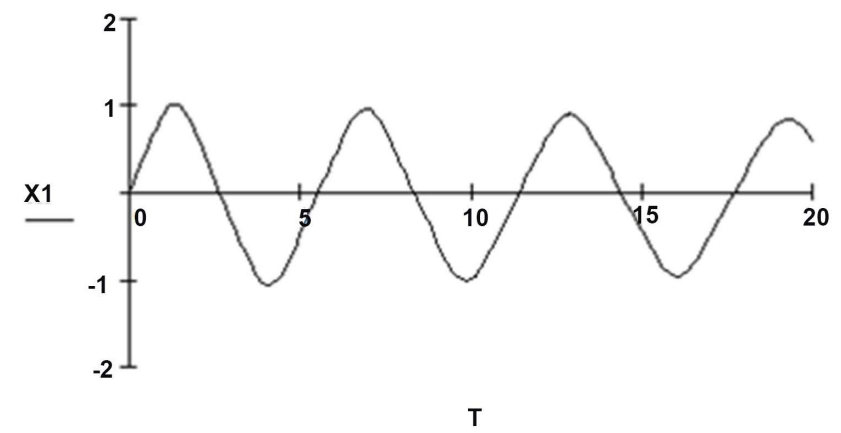

Figure 7. Oscillatory profile of Duffing equation for values $a=0.6, b=0.3, c=0.5$.

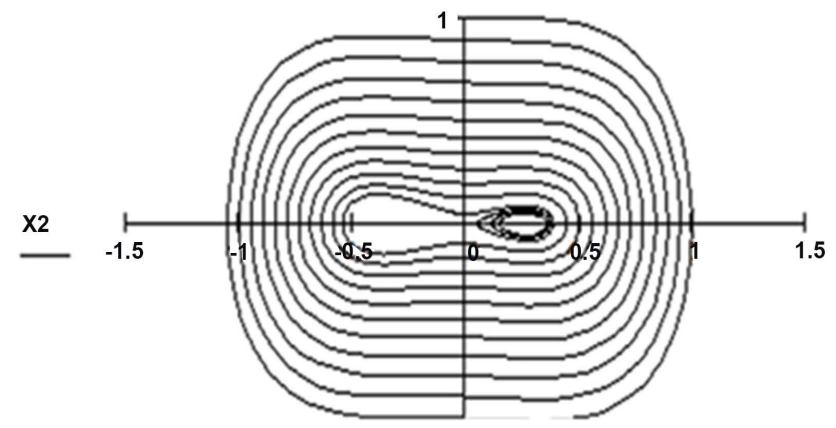

Figure 8. Phase portrait depicting the centre as an unstable node (parameters). 
method and Matcad has yielded successful results. We discovered that global stability of periodic solution was achieved through the construction of a suitable and complete Lyapunov function for the hard spring system using Cartwright method. From the above tables discussed and outlined, we could see that the stability behaviours of the solutions are similar despite the different methods. Our technique showed superiority above others because stability is a property of the equilibrium point and of the system.

\section{Conflicts of Interest}

The authors declare no conflicts of interest regarding the publication of this paper.

\section{References}

[1] Bender, C.M. and Orszag, S.A. (1999) Advanced Mathematical Method for Scientists and Engineers Asymptotic Methods and Perturbation Theory. Springer, New York, 545-551.

[2] Zeeman, E. (1976) Duffing Equation in Brain Modelling. Bulletin Institute of Mathematics and Its Applications, 12, 207-214.

[3] Oyesanya, M.O. (2008) Duffing Oscillator as Model for Predicting Earthquake Occurrence I. Journal of Nigerian Association of Mathematical Physics, 12, 133-142.

[4] Wang, G., Zheng, W. and He, S. (2002) Estimation of Amplitude and Phase of a Weak Signal by Using the Property of Sensitive Dependence on Initial Condition of a Non-Linear Oscillator. Signal Processing, 82, 103-115. https://doi.org/10.1016/S0165-1684(01)00166-9

[5] Yang, L. and Li, Y.K. (2014) Existence and Global Exponential Stability of Almost Periodic Solution for a Class of Delay Duffing Equation on Time Scale. Abstract and Applied Analysis, 2014, Article ID: 857161.

https://doi.org/10.1155/2014/857161

[6] Bhatti, M. and Lara M. (2008) Periodic Solutions of the Duffing Equation. Birkhäuser Verlag, Bassel, Switzerland.

[7] Sani, G. and Allain, H. (1989) N-Cyclic Function and Multiple Subharmonic Solutions of Duffing Equation.

[8] Ortega, R. (1994) Some Application of Topological Degree to Stability Theory in Topological Methods in Differential Equation and Inclusion. Journal of London Mathematical Society, 42, 505-516.

[9] Njoku, F.I. and Omari, P. (2003) Stability Properties of Periodic Solutions of a Duffing Equation in the Presence of Lower and Upper Solutions. Applied Mathematics and Computation, 135, 471-490. https://doi.org/10.1016/S0096-3003(02)00062-0

[10] Torres, P.J. (2004) Existence and Stability of Periodic Solutions of a Duffing Equation by Using a New Maximum Principle. Mediterranean Journal of Mathematics, 1, 470-486. https://doi.org/10.1007/s00009-004-0025-3

[11] Oyesanya, M.O. and Nwamba, J.I. (2013) Stability Analysis of Damped Cubic-Quintic Duffing Oscillator. World Journal of Mechanics, 3, 43-57. https://doi.org/10.4236/wjm.2013.31003

[12] Ezeilo, J.O.C. (1966) An Estimate for the Solution of a Certain System of Differen- 
tial Equations. Nigeria Journal of Science Association, 1, 5-10.

[13] Ezeilo, J.O.C. (1963) An Extension of a Property of the Phase Space Trajectories of a Third Order Differential Equation. Annali di Matematica Pura ed Applicata, 63, 387-397. https://doi.org/10.1007/BF02412186

[14] Afuwape, A.U. (1983) Ultimate Boundedness Result for a Certain System of Third Order Non-Linear Differential Equation. Journal of Mathematical Analysis and Application, 97, 140-150. https://doi.org/10.1016/0022-247X(83)90243-3

[15] Afuwape, A.U. (1983) Uniform Utimate Boundedness Result for Some Third Order Nonlinear Differential Equation. KTP, Trieste, preprint IC/90/405.

[16] Ogundare, S.A. (2009) Qualitative and Quantitative Properties of Solution of Ordinary Differential Equations. Ph.D. Thesis, University of Forte Hare, Alice, South Africa, 48-63.

[17] Nayfeh, A.H. and Mook, D.T. (1979) Nonlinear Oscillation. Wiley Publication, New York.

[18] Thomsen, J.J. (2003) Vibration and Stability. Advanced Theory, Analysis and Tools. Springer, Toronto, London, 273-281. https://doi.org/10.1007/978-3-662-10793-5

[19] Seyranian, A.P. and Wang, Y. (2011) On Stability of Periodic Solution of Duffing Equation. Journal of National Academy of Science of Armenia, 111, 211-232.

[20] Moon, F.C. and Holmes, P.J. (1979) Chaotic Oscillators: Theory and Applications. Journal of Sound and Vibration, 65, 275-296.

[21] Haung, J.H., Su, R., Khali, H.K. and Chen, S.H. (2009) Computers and Studies. International Journal of Mathematical Analysis, 87, 1624-1630.

[22] El-Bassiouny, A.F. and Abadel-Khalik, A. (2010) Evolution Equation. Physica Scripta, 81, 56-62.

[23] Cartwright, M.L. (1956) On the Stability of Solution of Certain Differential Equation of the Fourth Order. The Quarterly Journal of Mechanics and Applied Mathematics, 9, 185-194. https://doi.org/10.1093/qjmam/9.2.185

[24] Tiryaki, A. and Tunc, C. (1995) Construction of Lyapunov Function for Certain Fourth-Order Autonomous Differential Equation. Indian Journal of Pure Application of Mathematics, 26, 225-232.

[25] Delves, L.M. and Mohamed, J.L. (1985) Computer Methods for Integral Equations. Cambridge University Press, New York, 376. https://doi.org/10.1017/CBO9780511569609

[26] Hairer, Z., Ernst, J., Wanner, D. and Gerhard, G. (1991) Solving Ordinary Differential Equation. In: Springer Series in Computational Mathematics, Vol. 73, Springer-Verlag, Berlin, 273-289.

[27] Atkinson, F.A. (1955) On Second Order Non-Linear Oscillation. Pacific Journal of Mathematics, 5, 643-647. https://doi.org/10.2140/pjm.1955.5.643

[28] Boyd, P.J. (2000) Chebychev and Fourier Spectral Method Edition. Dover Publication Inc., New York, 360.

[29] Jordan, D.W. and Smith, P. (1977) Nonlinear Analysis and Differential Equations. Clavendon Press, Oxford.

[30] Thompson, J.M.T. and Stewart, H.B. (1986) Nonlinear Dynamics and Chaos. John Wiley \& Sons, New York.

[31] Puu, T. (2000) Attractors, Bifurcations and Chaos. Non-Linear Phenomena in Economics. 2nd Edition, Springer Verlag, Berlin Heidelberg, 465-469.

https://doi.org/10.1007/978-3-662-04094-2 
[32] Ueda, Y. (2000) Randomly Transitional Phenomena in the System Governed by Duffing's Equation. Journal of Statistical Physics, 20, 181-196. https://doi.org/10.1007/BF01011512

[33] Zhang, W.B. (2005) Differential Equations, Bifurcations and Chaos in Economics. 4th Edition, World Scientific Publishing, Hackensack, NJ, 231-243. https://doi.org/10.1142/5827

[34] Ezeilo, J.O.C and Ogbu, H.M. (2009) Construction of Lyapunov Type of Functions for Some Third Order Differential Equation by Method of Integration. Journal of Science Teaching Association of Nigeria, 45, 59-63.

[35] Wiggins, S.S. (1990) The Geometrical Point of View of Dynamical Systems: Background Material, Poincaré Maps, and Examples. In: Introduction to Applied Nonlinear Dynamical Systems and Chaos, Springer Verlag, New York, 5-192.

https://doi.org/10.1007/978-1-4757-4067-7_2 